\title{
Article \\ Newton's Third Law in the Framework of Special Relativity for Charged Bodies Part 2: Preliminary Analysis of a Nano Relativistic Motor
}

\author{
Asher Yahalom ${ }^{1,2}$ (D) \\ 1 Department of Electrical \& Electronic Engineering, Faculty of Engineering, Ariel University, Ariel 40700, \\ Israel; asya@ariel.ac.il; Tel.: +972-54-7740294 \\ 2 Center for Astrophysics, Geophysics, and Space Sciences (AGASS), Ariel University, Ariel 40700, Israel
}

check for

updates

Citation: Yahalom, A. Newton's Third Law in the Framework of Special Relativity for Charged Bodies Part 2: Preliminary Analysis of a Nano Relativistic Motor. Symmetry 2022, 14, 94. https://doi.org/ $10.3390 /$ sym 14010094

Academic Editors: Jacek Karwowski and Young S. Kim

Received: 19 November 2021

Accepted: 4 January 2022

Published: 7 January 2022

Publisher's Note: MDPI stays neutral with regard to jurisdictional claims in published maps and institutional affiliations.

Copyright: (c) 2022 by the authors. Licensee MDPI, Basel, Switzerland. This article is an open access article distributed under the terms and conditions of the Creative Commons Attribution (CC BY) license (https:/ / creativecommons.org/licenses/by/ $4.0 /)$.

\begin{abstract}
Background: In a recent paper discussing Newton's third law in the framework of special relativity for charged bodies, it was suggested that one can construct a practical relativistic motor provided high enough charge and current densities are available. As on the macroscopic scale charge density is limited by the phenomena of dielectric breakdown, it was suggested to take advantage of the high charge densities which are available on the microscopic scale. (2) Methods: We use standard physical theories such as Maxwell electrodynamics and quantum mechanics, supplemented by tools from vector analysis and numerics. (3) Results: We show that a hydrogen atom either in the ground state or excited state will not produce a relativistic engine effect, but by breaking the symmetry or putting the electron in a wave packet state may produce relativistic motor effect. (4) Conclusions: A highly localized wave packet will produce a strong relativistic motor effect. The preliminary analysis of the current paper suggests new promising directions of research both theoretical and experimental.
\end{abstract}

Keywords: relativity; nano technology; magnetic materials; hydrogen; symmetry breaking; relativistic engine

\section{Introduction}

Relativity describes space-time structure. In Einstein's famous 1905 article: “On the Electrodynamics of Moving Bodies" [1] the theory was introduced for the first time. The theory was derived both from empirical observations and the theory of electromagnetics, which was presented in the midst of the nineteenth century by Maxwell by his famous partial differential equations [2-4] which owe their modern formulation to Oliver Heaviside [5]. Those equations imply that an electromagnetic wave travels at the speed of light $c$, which led the scientific community to believe that light is electromagnetic. Albert Einstein [1] used this to formulate their theory of relativity, which postulated that $c$ is the maximal allowed speed in nature. According to the theory of relativity, any material body, message, signal (even if not electromagnetic), or field can not travel faster than light moving in vacuum. Thus, retardation is established, if a phenomena occurs at a distance $R$ from an observer, it will not be noticed for at least a time of $\frac{R}{c}$. This means that action and reaction cannot be generated simultaneously because of propagation speed.

Newton's laws of motion, laid the foundation for modern science. These laws describe the relationship between the acting forces and motion in response to those forces. Three laws were first written by Isaac Newton in his Philosophiae Naturalis Principia Mathematica, first published in 1687 [6,7]. Here, we are concerned only with the third law, which states: When one body exerts a force on another body, the second body simultaneously exerts a force equal in magnitude and opposite in direction on the first body. Due to Newton's third law, the sum of forces in a system which is not affected externally is zero. This law has a many experimental verifications and is a corner stone of physics. However, it is obvious that action and reaction cannot be generated at the same time because the speed of signal 
propagation is finite. Hence the third law cannot be correct, although it is valid for most practical applications due to high speed of signal propagation. Thus, the total sum of forces cannot be zero. Still in the quasi-static approximation retardation may be neglected as was noted by D'Abramo [8] and also in Section 3.1 of [9].

Current locomotive systems rely on interacting material parts; each gains momentum that is equal and opposite to the momentum obtained by its counterpart. A rocket ejects gas to propel itself forward. However, relativistic considerations suggest a new type of engine which is not derived from two material elements but of matter and field. It can be shown that the opposite amount of momentum to the one gained by the material body is gained by the field [10], thus the total momentum is indeed conserved. This is derived from a fundamental theorem due to Noether which requires that a physical system that is invariant under global translations will conserve its total linear momentum. A physical system which has both matter and field components is indeed invariant under global translations, while each component (either matter or field taken separately) is not invariant. For instance, if one translates the material but leave the field unchanged, obviously the charged particle will feel a different field value in there updated positions and thus will behave differently.

Feynman [4] has considered two charges moving orthogonally to each other, and showed that they contradict Newton's third law as forces that the charges induce do not cancel each other (last part of 26-2), this is solved in (27-6) in which Feynman noticed that the momentum gained by the two charge system is lost to the field, in the sense that the field acquires momentum of the same size and opposite direction as the two charge system.

We define a relativistic motor (engine) as a system in which its center of mass is in motion due to the interaction of its material components. Those parts may move with respect to each other or stay fixed to a rigid frame. This is of no consequence as are interests lie only in the center of mass propagation. We point out that a relativistic motor allows motion in three directions (vertical motion included), it does not contain any moving parts, it does not consume any type of fuel (and thus has zero carbon emission) it only transforms electromagnetic energy to kinetic energy, this type of energy may be supplied by solar panels and stored in batteries. Thus, it seems that the relativistic motor is perfect for space travel in which much of the space vehicle volume is devoted to fuel storage in the current technology. The energy can be put back into electromagnetic form once the vehicle reaches its destination indicating a very high potential efficiency.

Here it is assumed that the medium's magnetization and polarization are small and therefore we do not consider corrections to the Lorentz force described in [11]. Griffiths \& Heald [12] determined that the laws of Coulomb's and Biot-Savart describe the electric and magnetic field only for strictly static sources. In other cases, time-dependent generalizations of these laws as derived by Jefimenko [13] should be used. The expressions for the magnetic and electric field due to Jefimenko are used in the current paper and previous related work.

In an earlier work, Jefimenko's [3,13] equation was used to study the force between two loop currents [14]. This was later generalized to describe the forces between a permanent magnet and a current carrying loop [15]. As the device is forced for a finite duration, it will acquire momentum and energy in mechanical form. The question then arises if the law of momentum and energy conservation are violated. Linear momentum balance was discussed in [10]. The exchange of energy between the mechanical part of the relativistic engine and the electromagnetic field was studied in [16-18]. It was shown that the electromagnetic energy consumed is six fold the kinetic energy provided to the relativistic engine. It was also shown that energy might be radiated from the relativistic engine if the coils are misaligned.

Previous analysis assumed bodies that were macroscopically natural. In a recent paper [9] we abandoned this assumption and analyzed charged bodies, thus revealing the consequences of charge on a possible electric relativistic engine. The charged relativistic engine allows to maintain a finite momentum even if the current is not continuously increasing which is not the case for the uncharged engine. However, due to the phenomena of dielectric breakdown which enforces charge density maximal values and the limitations 
on current density that can be transferred even through a superconducting wire it is concluded that for any reasonable geometrical size the momentum that can be gained in a relativistic charged engine is quite modest.

It was thus suggested to take advantage of the high charge densities that are available in the microscopic realm, for example in ionic crystals. We will further pursue this idea in this paper in which we calculate the high charge densities and current densities in the atomic level. We will also deduce a preliminary form for the optimized wave function in terms of relativistic engine performance. We shall not derive the basic equations of the relativistic engine here the interested reader is referred to [9], only the principle results will be quoted.

\section{Main Results Regarding the Charged Relativistic Motor}

Let us consider two charged sub systems having the charge densities $\rho_{1}, \rho_{2}$ and current densities $\vec{J}_{1}, \vec{J}_{2}$ then according to the analysis presented in [9] the following force is acting on the physical system composed of those two sub systems:

$$
\vec{F}_{T}^{[2]}=\frac{\mu_{0}}{4 \pi} \partial_{t} \iint d^{3} x_{1} d^{3} x_{2}\left[\frac{1}{2}\left(\rho_{2} \partial_{t} \rho_{1}-\rho_{1} \partial_{t} \rho_{2}\right) \hat{R}-\left(\rho_{1} \vec{J}_{2}+\rho_{2} \vec{J}_{1}\right) R^{-1}\right]
$$

in the above $\mu_{0}=4 \pi 10^{-7}$ is the vacuum permeability in MKS units, $\partial_{t}$ is a partial temporal derivative. $\int d^{3} x_{1}$ and $\int d^{3} x_{2}$ are integrals of the volume of the first and second subsystems, respectively. We define $\vec{R}=\vec{x}_{1}-\vec{x}_{2}, R=|\vec{R}|, \hat{R}=\frac{\vec{R}}{R}$.

In the next subsections, we analyze examples of the implications of this formula. We remark that the it is based on a second order approximation, in some fast changing systems the second order correction will not be sufficient and higher order correction will be requited.

\subsection{Some Basic Preliminary Observations}

According to Newton's second law, a system with a non zero total force in its center of mass, must have a change in its total linear momentum $\vec{P}(t)$ :

$$
\vec{F}_{T}^{[2]}=\frac{d \vec{P}}{d t}
$$

It can be shown that gravity alone has no "force" in free space and in case where gravity has a considerable effect the right hand side should be replaced by a geodesic equation of motion. However, in most cases electromagnetism is orders of magnitude stronger. Hence the right hand side should be considered a valid approximation to the geodesic form. Assuming that $\vec{P}(-\infty)=0$ and null current and charge densities at the same time, it follows that:

$$
\vec{P}(t)=\frac{\mu_{0}}{4 \pi} \iint d^{3} x_{1} d^{3} x_{2}\left[\frac{1}{2}\left(\rho_{2} \partial_{t} \rho_{1}-\rho_{1} \partial_{t} \rho_{2}\right) \hat{R}-\left(\rho_{1} \vec{J}_{2}+\rho_{2} \vec{J}_{1}\right) R^{-1}\right]
$$

Thus, we obtain finite linear momentum for stationary charge and current densities:

$$
\vec{P}(t)=-\frac{\mu_{0}}{4 \pi} \iint d^{3} x_{1} d^{3} x_{2}\left(\rho_{1} \vec{J}_{2}+\rho_{2} \vec{J}_{1}\right) R^{-1}
$$

it follows that the charged relativistic engine can produce forward linear momentum without interacting with any external system except it own electromagnetic field. The above expression can be simplified using the instantaneous (non retarded) potentials:

$$
\Phi_{1}\left(\vec{x}_{2}\right)=\frac{1}{4 \pi \epsilon_{0}} \int d^{3} x_{1} \frac{\rho_{1}\left(\vec{x}_{1}, t\right)}{R}, \quad \Phi_{2}\left(\vec{x}_{1}\right)=\frac{1}{4 \pi \epsilon_{0}} \int d^{3} x_{2} \frac{\rho_{2}\left(\vec{x}_{2}, t\right)}{R}
$$


$\epsilon_{0}=8.8510^{-12}$ is the vacuum permittivity in MKS units. Using Equation (5) we may write:

$$
\vec{P}(t)=-\frac{1}{c^{2}}\left[\int d^{3} x_{2} \Phi_{1} \vec{J}_{2}+\int d^{3} x_{1} \Phi_{2} \vec{J}_{1}\right]
$$

in which we are reminded that $\mu_{0} \epsilon_{0}=\frac{1}{c^{2}}$. Another important result is that in a charged relativistic motor we do not need both subsystems to be charged, that is we can take $\rho_{2}=0$ :

$$
\vec{P}(t)=-\frac{\mu_{0}}{4 \pi} \iint d^{3} x_{1} d^{3} x_{2} \rho_{1} \vec{J}_{2} R^{-1}=-\frac{1}{c^{2}} \int d^{3} x_{2} \Phi_{1} \vec{J}_{2}
$$

provided that the system has a non vanishing current density $\vec{J}_{2}$. The same result will be obtained if sub systems 1 and 2 are charged but system 1 lacks current density: $\vec{J}_{1}=0$.

In Reference [10] we showed that the forward linear momentum gained by the mechanical system will be nullified by a backward linear momentum gained by the electromagnetic field. We shall now consider the challenges involved in obtaining a practical charged relativistic motor.

\subsection{Charge Density Limitations}

The amount of charge that can be concentrated in a volume or a surface is limited due to electrical breakdown. When electric breakdown occurs the neutral medium is separated into electron and ions and becomes conducting. A discharge is dictated and the charge density is nullified. A typical dielectric strength $E_{\max }$ of air is about $3 \mathrm{MV} / \mathrm{m}$ [19], for high vacuum one can achieve a dielectric strength of 20-40 MV/m [20] and a diamond has a dielectric strength of $2000 \mathrm{MV} / \mathrm{m}$ [21]. For an infinite charged surface the surface density $\sigma$ is related to the electric field according to:

$$
\sigma=2 \epsilon E<\sigma_{\max }=2 \epsilon E_{\max }, \quad \epsilon=\epsilon_{r} \epsilon_{0}
$$

in which $\epsilon_{r}$ is the relative susceptibility of the material encapsulating the surface. For air we thus obtain $\sigma_{\max } \simeq 53 \mu \mathrm{C} / \mathrm{m}^{2}$. We are now interested in calculating the charge $Q$ which one can concentrate in a given volume. Notice that for a spherical symmetric charged ball we obtain a radial field $E$ which takes the value:

$$
E=\frac{k Q}{r^{2}}, \quad k=\frac{1}{4 \pi \epsilon_{0}}
$$

at a distance $r$. Thus, the field is strongest on the surface of the ball itself, for which $r=r_{s}$. It follows that:

$$
\frac{k Q}{r_{s}^{2}}<E_{m} \Rightarrow Q<Q_{m}=\frac{1}{k} r_{s}^{2} E_{m}
$$

For a size of $1 \mathrm{~m}$, the maximal charge is $3.310^{-4} \mathrm{C}$. Hence, regardless if we have surface charge or a volume charge the maximal charge scales as the square of the dimension of the system. A plausible approach to enlarge charge density is by using an electret. Fluorinated parylene (parylene HT, SCS) has a remarkable surface charge density of $3.7 \mathrm{mC} / \mathrm{m}^{2}$ obtained for a $7.3 \mu \mathrm{m}$ film [22]. This material also has a dielectric strength of $204.58 \mathrm{MV} / \mathrm{m}$. Notice, however, that as the thickness of this material grows the charge density is reduced.

\subsection{Macroscopic Current Density Limitations}

The current a device can generate depends on its voltage difference and internal resistance. Provided that the external impedance is insignificant, the resulting currents become short currents as large as a few thousand amperes for a standard domestic electrical installation and as large as hundreds of thousands amperes for an industrial installation. Current flowing through a resistive conductor will cause heat generation. High currents thus require a thick conductor to avoid over heating. This deficiency can be removed using a superconductor, however, this solution will require cooling to low temperatures, making 
the system much more cumbersome. Notice, however, that even a superconducting material is limited by a critical current density, above which the superconducting properties are lost. Jung et al. [23] have obtained significant critical current densities which are about $5 \mathrm{kA} / \mathrm{cm}^{2}$. We point out that coil windings will enable to reuse the current, and thus the winding number of coil passing through a given area is critical for optimal performance. Proximity of current and charge will affect the amount of generated linear momenta according to Equation (7). However, installing a conductor close to the charge may result in discharge, hence a balance is required.

\subsection{Scalability}

It is obvious from Equation (7) that a larger relativistic motor is more powerful. However, since the charge surface density is limited the linear momentum gained will scale as the second power of the dimension of the charged subsystem and as the third power of the dimension of the current carrying system.

\subsection{Energy}

A motor of mass $M$ will have a kinetic energy of:

$$
E_{k}=\frac{\vec{P}^{2}}{2 M}
$$

$\vec{P}$ is calculated using Equation (3). The energy needed for the actual operation of the motor may be greater or equal to the kinetic energy of the engine even in ideal cases in which loss mechanisms such as drag, friction and ohmic resistance are of no consequence. For an uncharged relativistic engine the energy cost is $E=6 E_{k}$ [18], it follows that the energy needed by a charged relativistic engine is of the order of magnitude of the kinetic energy calculated in Equation (11) (but larger). The power needed is the time derivative of the energy, and thus it follows from Equation (3) that it will depend on current rise time. To conclude, we point out that in ideal conditions in which drag losses, viscosity losses (space travel) and ohmic losses (superconducting wires) may be neglected the efficiency of the relativistic engine is infinite, and the mechanical energy can be recovered as the engine comes to a full stop. This approach is partially implemented in today hybrid vehicles, in which pressing the brakes converts the kinetic energy of the car to energy stored in a battery.

Notice that if not aligned correctly a relativistic motor may loss energy by radiation. This was demonstrated in previously for an uncharged relativistic engine $[17,18]$. The total force generation given by Equation (1) includes also radiating fields, hence it will not affect the current results.

\section{The Nano Relativistic Engine}

The following section is similar to Section 5 of [9] and is repeated here for completeness. In Reference [9] we have shown that intrinsic parameter limitations, especially dielectric breakdown lead to somewhat modest amount of momenta that can be gained by relativistic motor. "However, it seams that in the microscopic domain this limitation is rather relaxed. For example, if consider ionic crystals such as the prevalent table salt: $\mathrm{Na}^{+} \mathrm{Cl}^{-}$. This salt solidifies to a face centered cubic lattice in which the lattice constant is $l=564.02 \mathrm{pm}$. Taking for example the 100 plane of this lattice (see Figure 1) we can see that the charge density is periodical in which in each half unit cell we have a surface charge density of \pm 2 Coulomb $/ \mathrm{m}^{2}$. This is of course thousand time larger than available macroscopic charge densities (see Section 2.2), however, on the macroscopic average this leads to a null charge density. We also notice that a smaller lattice constant will cause a still higher charge density, but it seems that the lattice constant cannot be smaller than the relevant atom hence it is bounded from below. 


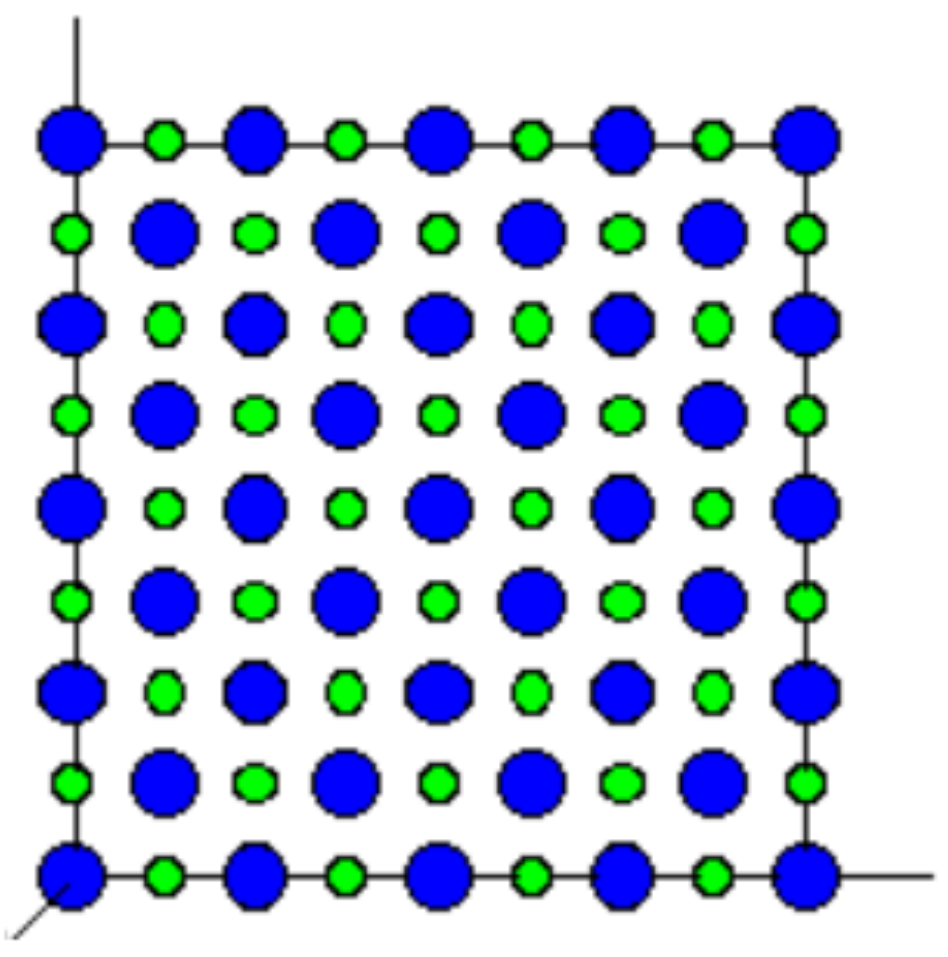

Figure 1. Depicted is the 100 plane of a lattice of table salt $\mathrm{Na}^{+} \mathrm{Cl}^{-}$, blue circles are Sodium positive ions and yellow circles are chlorine negative ions.

To see how we can avoid this unfortunate reality we will study Equation (7), using a spatial Fourier transform of both the scalar potential and the current density given by:

$$
\begin{aligned}
\Phi_{1}(\vec{k}) & =\int_{-\infty}^{+\infty} \int_{-\infty}^{+\infty} \int_{-\infty}^{+\infty} \Phi_{1}(\vec{x}) e^{-i \vec{k} \cdot \vec{x}} d^{3} x \\
\vec{J}_{2}(\vec{k}) & =\int_{-\infty}^{+\infty} \int_{-\infty}^{+\infty} \int_{-\infty}^{+\infty} \vec{J}_{2}(\vec{x}) e^{-i \vec{k} \cdot \vec{x}} d^{3} x
\end{aligned}
$$

Using the theorem of Parseval [24], we may now write Equation (7) in the form:

$$
\vec{P}(t)=-\frac{1}{c^{2}(2 \pi)^{3}} \int_{-\infty}^{+\infty} \int_{-\infty}^{+\infty} \int_{-\infty}^{+\infty} d^{3} k \Phi_{1}^{*}(\vec{k}) \vec{J}_{2}(\vec{k})
$$

In this form it is obvious, that a microscopic distribution of periodic charge density will be beneficial if it is a accompanied by a current density distribution of the same period. How can we obtain such a microscopic charge density distribution? To this end we remind the reader that microscopic currents are associated with the electronic motion and electronic spin. The magnetization $\vec{M}$ is related to the magnetization current $\vec{J}_{M}$ by the formulae [15]:

$$
\vec{J}_{M} \equiv \vec{\nabla} \times \vec{M}
$$

We may replace in Equations (6), (7) and (13) $\vec{J}$ by $\vec{J}_{M}$ to obtain a similar effect. Furthermore, the magnetization $\vec{M}$ is related to a the microscopic dipole moments $\vec{m}_{i}$ through:

$$
\vec{M} \equiv \frac{1}{V} \sum_{i} \vec{m}_{i}
$$

in the above we sum over all dipoles and divide by the total sample volume $V$. In known magnetic materials such as iron, magnetic dipole moments are a result of atom spin configuration. For instance, for $\alpha$-iron the spins of two unpaired electrons in each individual atom align with the spins of its neighbors. This follows as the orbitals of those electrons $\left(d_{z^{2}}\right.$ and $\left.d_{x^{2}-y^{2}}\right)$ do not 
point at the prescribed direction towards neighboring atoms in the lattice, and as this is the case are not involved in bonding. We thus suggest that the structure of a relativistic engine will include an ionic lattice in which one species of atom (say the positively charged) will involve free spins that may be manipulated by an external magnetic field thus creating a relativistic motor which can be easily manipulated in all axis." In the following section we study some manifestations of a relativistic engine in the atomic scale.

\section{Relativistic Engine in the Microscopic Scale}

In the microscopic scale relevant to the relativistic engine to be discussed here matter is composed of electron and nuclei which are put together to become natural atoms, ions, molecules and lattice structures. There are of course smaller scales which one can discuss which are relevant to nuclear physics, elementary particles etc. but this will not concern us here. Matter at the microscopic scale is described by quantum mechanics. In this preliminary work it will suffice to discuss a one electron system in which the nuclei effecting the motion of the electron are modeled as point charges.

\subsection{Schrödinger's Electron}

Schrödinger's electron is an electron in which the spin property is ignored. The earliest appearance of the non-relativistic probability density and current density satisfying a continuity equation is due to Schrödinger himself [25], obtained from his time-dependent wave-equation:

$$
i \hbar \dot{\psi}=\hat{H} \psi, \quad \hat{H}=-\frac{\hbar^{2}}{2 m} \vec{\nabla}^{2}+V
$$

here $i=\sqrt{-1}$ and $\psi$ is a complex function. $\dot{\psi} \equiv \frac{\partial \psi}{\partial t}$ is the partial time derivative of the complex wave function. $\hbar=\frac{h}{2 \pi}$ denotes Planck's constant divided by $2 \pi$ and $m$ is the mass, $V$ is the potential function. We introduce the modulus $a$ and phase $\phi$ through:

$$
\psi=a e^{i \phi}
$$

The following continuity equation is satisfied provided that $\psi$ satisfies Equation (16):

$$
\frac{\partial \tilde{\rho}}{\partial t}+\vec{\nabla} \cdot \vec{J}_{p}=0
$$

in which the probability density is defined as:

$$
\tilde{\rho}=a^{2}
$$

and is of course normalized, that is:

$$
\int \tilde{\rho} d^{3} x=1
$$

The probability current density is:

$$
\vec{J}_{p}=\frac{\hbar}{m} \tilde{\rho} \vec{\nabla} \phi
$$

As the charge density of an electron with charge $-e$ is:

$$
\rho=-e \tilde{\rho}
$$

it follows that the current density is:

$$
\vec{J}=-e \vec{J}_{p}=-\frac{\hbar e}{m} \tilde{\rho} \vec{\nabla} \phi=\frac{\hbar}{m} \rho \vec{\nabla} \phi,
$$


Satisfying the charge density continuity equation:

$$
\frac{\partial \rho}{\partial t}+\vec{\nabla} \cdot \vec{J}=0
$$

\subsection{Pauli's Electron}

Schrödinger's quantum mechanics is limited to the description of spin less particles. If spin is to be taken into account we must use the Pauli equation (for a non-relativistic particle):

$$
i \hbar \dot{\psi}=\hat{H} \psi, \quad \hat{H}=-\frac{\hbar^{2}}{2 m}\left[\vec{\nabla}-\frac{i e}{\hbar c} \vec{A}\right]^{2}+\mu \vec{B} \cdot \vec{\sigma}+V
$$

in this context $\psi$ is a two dimensional complex column vector (a spinor), $\hat{H}$ is now a two dimensional hermitian operator matrix, $\mu$ describes the magnetic moment. The electromagnetic interaction is given through $\vec{A}$ and $V$ potentials and the magnetic field $\vec{B}=\vec{\nabla} \times \vec{A} . \vec{\sigma}$ is a vector of Pauli matrices as follows:

$$
\sigma_{1}=\left(\begin{array}{cc}
0 & 1 \\
1 & 0
\end{array}\right), \quad \sigma_{2}=\left(\begin{array}{cc}
0 & -i \\
i & 0
\end{array}\right), \quad \sigma_{3}=\left(\begin{array}{cc}
1 & 0 \\
0 & -1
\end{array}\right)
$$

A spinor $\psi$ satisfying Equation (25) will also satisfy a continuity equation:

$$
\frac{\partial \tilde{\rho}}{\partial t}+\vec{\nabla} \cdot \vec{J}_{p}=0
$$

In the above:

$$
\tilde{\rho}=\psi^{\dagger} \psi, \quad \vec{J}_{p}=\frac{\hbar}{2 m i}\left[\psi^{\dagger} \vec{\nabla} \psi-\left(\vec{\nabla} \psi^{\dagger}\right) \psi\right]-\frac{e}{m c} \vec{A} \tilde{\rho} .
$$

$\psi^{\dagger}$ describes a row vector (the transpose) whose components are the complex conjugate of $\psi$. Holland [26] has derived the following spinor representation:

$$
\psi=R e^{i \frac{\chi}{2}}\left(\begin{array}{c}
\cos \left(\frac{\theta^{\prime}}{2}\right) e^{i \frac{\phi^{\prime}}{2}} \\
i \sin \left(\frac{\theta^{\prime}}{2}\right) e^{-i \frac{\phi^{\prime}}{2}}
\end{array}\right) \equiv\left(\begin{array}{c}
\psi_{\uparrow} \\
\psi_{\downarrow}
\end{array}\right) .
$$

Thus, the probability density is:

$$
\tilde{\rho}=\psi^{\dagger} \psi=R^{2} \Rightarrow R=\sqrt{\tilde{\rho}} .
$$

Furthermore, the charge density is:

$$
\rho=-e \tilde{\rho}=-e \psi^{\dagger} \psi=-e R^{2} .
$$

The spin density derived from the representation given in Equation (29) is:

$$
\hat{s} \equiv \frac{\psi^{+} \vec{\sigma} \psi}{\rho}=\left(\sin \theta^{\prime} \sin \phi^{\prime}, \sin \theta^{\prime} \cos \phi^{\prime}, \cos \theta^{\prime}\right)
$$

This gives an obvious physical interpretation to $\theta^{\prime}, \phi^{\prime}$ as angles which describe the projections of the spin density. $\theta^{\prime}$ is the elevation angle and $\phi^{\prime}$ is the azimuthal angle. The probability current density can now be derived by inserting $\psi$ into Equation (28) thus obtaining the expression:

$$
\vec{J}_{p}=\frac{\hbar}{2 m} \tilde{\rho}\left(\vec{\nabla} \chi+\cos \theta^{\prime} \vec{\nabla} \phi^{\prime}\right)-\frac{e}{m c} \tilde{\rho} \vec{A}
$$


if the vector potential contribution is not significant, we may write:

$$
\vec{J}_{p}=\frac{\hbar}{2 m} \tilde{\rho}\left(\vec{\nabla} \chi+\cos \theta^{\prime} \vec{\nabla} \phi^{\prime}\right) .
$$

Hence, the electric current density is:

$$
\vec{J}=-e \vec{J}_{p}=-\frac{e \hbar}{2 m} \tilde{\rho}\left(\vec{\nabla} \chi+\cos \theta^{\prime} \vec{\nabla} \phi^{\prime}\right)=\frac{\hbar}{2 m} \rho\left(\vec{\nabla} \chi+\cos \theta^{\prime} \vec{\nabla} \phi^{\prime}\right) .
$$

If the electron is in a definitive spin state (say spin up) and the vector potential is null, Equation (25) is the same as Equation (16). Thus, for preliminary order of magnitude estimations Schrödinger equation solutions will suffice.

\subsection{The Hydrogen Atom}

The hydrogen atom is one of the simplest quantum mechanical systems, and one of the few quantum mechanical systems that can be solved exactly. The hydrogen atom is composed of a proton and an electron, the proton is modeled as a positive point charge of charge $+e$ located conveniently at the origin of the axis. $V$ in Equation (16) is thus equal to $-e \Phi$ which is felt by an electron of charge $-e$, in which $\Phi$ is given in Equation (5) for a positive point charge $+e$ located at the origin of axis, that is:

$$
V=-e \Phi=-\frac{k e^{2}}{r} .
$$

The electron wave function can be of the form:

$$
\psi=e^{-\frac{i E_{n} t}{\hbar}} \psi_{n}, \quad \hat{H} \psi_{n}=E_{n} \psi_{n}
$$

In which $\psi_{n}$ is a spatial Eigenfunction of the Hamiltonian $\hat{H}$ with Eigenenergy $E_{n}$. The electron can be in a definite energy state or in a superposition of states. The functional form of $\psi_{n}$ is well known and is given in terms of the spherical coordinates $r, \theta, \varphi$ as follows:

$$
\psi_{n l m}=\sqrt{\left(\frac{2}{n a_{0}}\right)^{3} \frac{(n-l-1) !}{2 n(n+l) !}} e^{-\frac{r^{\prime}}{2}} r^{\prime l} L_{n-l-1}^{2 l+1}\left(r^{\prime}\right) Y_{l}^{m}(\theta, \varphi) .
$$

In the above $L_{n-l-1}^{2 l+1}$ is the generalized Laguerre polynomial of degree $n-l-1$, and $Y_{l}^{m}(\theta, \varphi)$ is a spherical harmonic function of degree $l$ and order $m$ defined as:

$$
Y_{l}^{m}(\theta, \varphi)=(-1)^{m} \sqrt{\frac{2 l+1}{4 \pi} \frac{(l-m) !}{(l+m) !}} P_{l}^{m}(\cos \theta) e^{i m \varphi} .
$$

In which $P_{l}^{m}$ are associated Legendre polynomials. The definition also contains the reduced Bohr radius:

$$
a_{0} \equiv \frac{4 \pi \epsilon_{0} \hbar^{2}}{m_{e}^{\prime} e^{2}} \simeq \frac{\hbar^{2}}{k m_{e} e^{2}} \simeq 0.5310^{-10} \mathrm{~m}, \quad m_{e}^{\prime} \equiv \frac{m_{e} m_{p}}{m_{e}+m_{p}} \simeq m_{e}
$$

in which $m_{e}$ and $m_{p}$ are the masses of the electron and proton, respectively. Finally we use a normalized radial coordinate $r^{\prime}$ defined as:

$$
r^{\prime} \equiv \frac{2 r}{n a_{0}} .
$$

The Eigen energies defined in Equation (37) are functions of the principal quantum number $n=1,2,3 \ldots$ :

$$
E_{n}=-\frac{m_{e}^{\prime} e^{4}}{32 \pi^{2} \epsilon_{0}^{2} \hbar^{2}} \frac{1}{n^{2}}
$$


Hence the function $\psi_{n l m}$ are degenerate in the sense that different functions have the same energy, the degeneracy can be lifted if there is a perturbation that changes the potential $V$ to a form that is not spherically symmetric. For a given energy the different Eigen functions are listed by their azimuthal quantum number $l=0,1,2, \ldots, n-1$ and their magnetic quantum number $m=-l, \ldots, l$. Notice that the amplitude of the wave function given in Equation (37) is a function of $r$ and $\theta$ only, but not of $\varphi$ and the time $t$.

$$
a(r, \theta)=|\psi|=\left|\psi_{n l m}\right| \Rightarrow \rho=-e\left|\psi_{n l m}\right|^{2} .
$$

Notice, also that the phase is a function of time and $\varphi$ but not of $r$ and $\theta$ :

$$
\phi(\varphi, t)=m(\varphi+\pi)-\frac{E_{n} t}{\hbar} .
$$

We are now in a position to calculate the current density given in Equation (23)

$$
\vec{J}=\frac{\hbar}{m_{e}} \rho \vec{\nabla} \phi=-m \frac{e \hbar}{m_{e}}\left|\psi_{n l m}\right|^{2} \frac{\hat{\varphi}}{r \sin \theta}
$$

(the reader should not confuse the magnetic number $m$ with the mass $m$ used in previous sections). In the above we have used the spherical representation of the nabla operator which is given in terms of the unit vectors $\hat{r}, \hat{\theta}, \hat{\varphi}$ as:

$$
\vec{\nabla}=\hat{r} \frac{\partial}{\partial r}+\frac{\hat{\theta}}{r} \frac{\partial}{\partial \theta}+\frac{\hat{\varphi}}{r \sin \theta} \frac{\partial}{\partial \varphi} .
$$

We notice that the current density is linear in the magnetic number $m$, in particular if $m=0$ there is no current density and thus no relativistic motor effect. We conclude that for an isolated hydrogen in the ground state $n=1, l=0, m=0$ there is no relativistic motor effect. However, also in excited states in which the current density does not necessarily vanish there will be no relativistic motor effect if the potential acting on the electron is spherically or cylindrically symmetric as is evident from Equation (7). To see this notice that:

$$
\hat{\varphi}=-\sin \varphi \hat{x}+\cos \varphi \hat{y}
$$

$\hat{x}$ and $\hat{y}$ are constant unit vectors in the $x$ and $y$ directions, respectively. In spherical coordinates the volume element is:

$$
d^{3} x=r^{2} \sin \theta d r d \theta d \varphi
$$

since $\Phi\left|\psi_{n l m}\right|^{2}$ is cylindrically symmetric it does not depend on $\varphi$ and the result follows immediately. We also notice that apparent singular terms in the current density of the form $\frac{1}{r \sin \theta}$ (see Equation (45)) are cancelled out by identical terms in the volume element.

How can we use an hydrogen atom as a component in a relativistic motor despite the fact that it is useless either in the ground state or in an excited state? In the following section we will suggest two approaches, in one the electron is not in an energy Eigen state but in a superposition of states and in the other the potential is not cylindrically symmetric. Both approaches will yield a finite relativistic engine effect.

However, before we proceed we make some orders of magnitude estimates to justify our endeavour. The volume charge density of an electron in a hydrogen atom has an order of magnitude:

$$
\bar{\rho}=\frac{e}{\frac{4}{3} \pi a_{0}^{3}} \simeq 2.610^{11} \text { Coloumb } / \mathrm{m}^{3} .
$$

The surface charge density of an electron in a hydrogen atom has an order of magnitude:

$$
\bar{\sigma}=\frac{e}{4 \pi a_{0}^{2}} \simeq 4.5 \text { Coloumb } / \mathrm{m}^{2} .
$$


this is of the same order of magnitude as we obtained for the salt crystal in Section 3, and a thousand times larger than what is available for macroscopic charge densities (see Section 2.2). The order of magnitude of the current density is:

$$
\bar{J}=\frac{e \hbar}{m_{e} a_{0}^{4}} \simeq 2.310^{18} \text { Ampere } / \mathrm{m}^{2}=2.310^{14} \text { Ampere } / \mathrm{cm}^{2}
$$

This current density is ten orders of magnitude larger than what can be achieved in a macroscopic scale (see Section 2.3). Although in the Copenhagen interpretation of quantum mechanics an electron does not have a trajectory and therefore does not have a velocity we can still associate with the current density a typical velocity:

$$
\bar{v}=\frac{\bar{J}}{\bar{\rho}} \sim 9.110^{6} \mathrm{~m} / \mathrm{s}=0.03 \mathrm{c}
$$

Hence the associated velocity is rather large although still significantly smaller than the speed of light.

\subsection{A Wave Packet of Hydrogen Atom Eigenstates}

Let us assume an idealized wave packet of the form:

$$
\psi=A e^{i k^{\prime} x}, \quad A=\left\{\begin{array}{cc}
\sqrt{\tilde{\rho}_{c}} & r<R_{\max } \\
0 & r \geq R_{\max }
\end{array}\right.
$$

in the above $k^{\prime}$ and $\tilde{\rho}_{c}$ are constants. As the wave function must be normalized it follows from Equation (20) that $\tilde{\rho}_{c}$ must take the following value:

$$
\tilde{\rho}_{c}=\frac{3}{4 \pi} R_{\max }^{-3}
$$

hence this wave function has a linear phase and a uniform amplitude which is confined inside a sphere of radius $R_{\max }$. It is certainly not an Eigen state of the hydrogen atom Hamiltonian but may be approximated by a superposition of the Eigenstates:

$$
\psi \simeq \sum_{n l m} a_{n l m}(t) \psi_{n l m}
$$

The desired functions $a_{n l m}(t)$ can be calculated using the orthonormality properties of the Eigen functions:

$$
a_{n l m}(t)=<\psi_{n l m} \mid \psi>=\int d^{3} x \psi_{n l m}^{*} \psi
$$

the preparation of such a state will require a suitable electromagnetic pulse in accordance with Equation (25), the shape of the pulse for creating and maintaining the wave packet is beyond the scope of the current paper.

We can now calculate the current density using Equation (23) as follows:

$$
\vec{J}=-\frac{\hbar e}{m_{e}} \tilde{\rho} \vec{\nabla} \phi=-\frac{\hbar k e}{m_{e}} \tilde{\rho}_{c} \hat{x}=-e v \tilde{\rho}_{c} \hat{x} \quad \text { for } \quad r<R_{\max }, \quad v \equiv \frac{\hbar k^{\prime}}{m_{e}},
$$

$v$ has the units of velocity. Let us now take system 1 in Equation (7) to be the proton and system 2 to be electron. The proton is conveniently modeled as a point charge located at the origin of axis. That is:

$$
\rho_{1}=e \delta^{(3)}\left(\vec{x}_{1}\right)
$$

It thus follows:

$$
\vec{P}(t)=-\frac{\mu_{0}}{4 \pi} \iint d^{3} x_{1} d^{3} x_{2} \rho_{1} \vec{J}_{2} R^{-1}==-\frac{\mu_{0} e}{4 \pi} \int d^{3} x_{2} \vec{J}_{2} r_{2}^{-1} .
$$


Plugging Equations (48) and (57) into the above expression and integrating will result in:

$$
\vec{P}(t)=\frac{1}{2} \mu_{0} e^{2} v \tilde{\rho}_{c} R_{\max }^{2} \hat{x}
$$

Taking into account normalization according to Equation (54) we obtain the following expression for $\vec{P}(t)$ :

$$
\vec{P}(t)=\frac{3}{8 \pi} \mu_{0} e^{2} v R_{\text {max }}^{-1} \hat{x} .
$$

It follows that the momentum gained by a relativistic motor is linearly proportional to the electron's "velocity" $v$ and inversely proportional to the wave packet spatial extension $R_{\text {max }}$. As the maximal speed generated by the hydrogen relativistic motor is obtained when the engine is not loaded and need to carry only its own mass it follows that:

$$
v_{\max }=\frac{P}{m_{p}}=\frac{3}{8 \pi m_{p}} \mu_{0} e^{2} v R_{\max }^{-1} .
$$

the electron mass $m_{e}$ is ignored as it is much smaller than the proton mass $m_{p}$. Thus, to obtain a predefined velocity $v_{\max }$ we need a wave packer of the radius:

$$
R_{\max }=\frac{3 \mu_{0} e^{2} v}{8 \pi m_{p} v_{\max }} .
$$

According to Equation (52) the typical "velocity" of the electron is $0.03 c$ in the hydrogen atom. Here we will assume that $v \simeq c$ for the purpose of obtaining the maximal $R_{\max }$ required, taking into account that in reality we will need a smaller $R_{\max }$ to achieve the desired velocity. We also remark that for a relativistic electron the Schrödinger formalism is not adequate and one should use a Dirac equation instead. Moreover, the relativistic engine considered so far assumes slow moving components where the relativistic effect is due to the retardation of the electromagnetic signal, a different mathematical treatment is needed if the components of the engine move with relativistic speeds. Taking this into account we make the following preliminary observations: for a typical car a velocity of $v_{\max }=50 \mathrm{~m} / \mathrm{s}=180 \mathrm{~km} / \mathrm{h}$ we obtain:

$$
R_{50} \simeq 1.410^{-11} \mathrm{~m}=0.26 a_{0}
$$

hence the wave packet is about quarter of the atomic size. If we need the hydrogen relativistic engine to reach the earth's escape velocity of $v_{\max }=11.2 \mathrm{~km} / \mathrm{s}$ it follows that:

$$
R_{\text {escape velocity }} \simeq 6.110^{-14} \mathrm{~m} \simeq 10^{-3} a_{0} \simeq 73 r_{p}
$$

in which $r_{p}=8.410^{-16} \mathrm{~m}$ is the proton charge radius. Thus, for such velocities the wave packet is of a typical nuclear size rather than an atomic size. Finally if we imagine that the relativistic engine will reach the maximal speed available in a Lorentzian space-time for a particle which is initially subluminal that is $v_{\max } \simeq c$ it follows that:

$$
R_{\text {light speed }} \simeq 2.310^{-18} \mathrm{~m} \simeq 310^{-3} r_{p}
$$

that is the wave packet must be of sub nuclear dimensions.

We conclude this section by calculating the standard deviation of the wave packet defined in Equation (53).

$$
\sigma_{x}^{2}=\int d^{3} x(x-E[x])^{2}|\psi|^{2}=\int d^{3} x(x-\bar{x})^{2} A^{2}
$$


In which the expectation value of position is:

$$
E[x]=\int d^{3} x x|\psi|^{2}=\int d^{3} x x A^{2}=0
$$

It follows thus that:

$$
\sigma_{x}^{2}=\int d^{3} x x^{2} A^{2}=\frac{1}{5} R_{\max }^{2}, \Rightarrow \sigma_{x}=\frac{1}{\sqrt{5}} R_{\max }
$$

Similarly:

$$
E[y]=E[z]=0, \quad \sigma_{y}=\sigma_{z}=\frac{1}{\sqrt{5}} R_{\max }
$$

Thus, the electron is expected to be found in coincidence with the proton with a standard deviation which is about half the size of the wave packet. Calculating the expectation value for the momentum operators $\hat{p}_{x}=-i \hbar \frac{\partial}{\partial x}$ we find that:

$$
E\left[p_{x}\right]=\int d^{3} x \psi^{*} \hat{p}_{x} \psi=-i \hbar \int d^{3} x \psi^{*} \frac{\partial \psi}{\partial x}=\hbar k^{\prime}
$$

similarly:

$$
\begin{aligned}
& E\left[p_{y}\right]=\int d^{3} x \psi^{*} \hat{p}_{y} \psi=-i \hbar \int d^{3} x \psi^{*} \frac{\partial \psi}{\partial y}=0, \\
& E\left[p_{y}\right]=\int d^{3} x \psi^{*} \hat{p}_{y} \psi=-i \hbar \int d^{3} x \psi^{*} \frac{\partial \psi}{\partial y}=0
\end{aligned}
$$

hence the expected velocity of the electron is equal to the current velocity defined in Equation (57) and in the same direction:

$$
v=\frac{E\left[p_{x}\right]}{m_{e}}=\frac{\hbar k^{\prime}}{m_{e}}
$$

The standard deviation of the momentum operator in any direction is infinite due to the ideal discontinues form of the wave function, meaning that when measuring the electron velocity any value can be obtained with non vanishing probability. We notice, however, that even for a more realistic and smooth wave packet we expect a non vanishing standard deviation of the electron momenta due to the Heisenberg uncertainty relation:

$$
\sigma_{p_{x}} \geq \frac{\hbar}{2 \sigma_{x}} \simeq \frac{\hbar}{R_{\max }}, \quad \sigma_{p_{y}} \geq \frac{\hbar}{2 \sigma_{y}} \simeq \frac{\hbar}{R_{\max }}, \quad \sigma_{p_{z}} \geq \frac{\hbar}{2 \sigma_{z}} \simeq \frac{\hbar}{R_{\max }} .
$$

hence the measured velocity of the electron may differ significantly from the expected current velocity.

\subsection{Symmetry Breaking}

As pointed out earlier the relativistic engine effect will be null for an hydrogen atom in either the ground or excited state due to the symmetry of the proton potential. However, if the symmetry is broken the relativistic engine effect is recovered. We thus assume an additional proton located at a distance $d$ from the hydrogen atom. This proton will break the cylindrical symmetry. In what follows we will consider system 1 as the additional proton and system 2 will be the electron. We will assume that the proton is a distance $d$ from the axis origin in the negative $x$ direction. The potential generated by this additional proton locate at $\vec{x}_{1}=-d \hat{x}$ is according to Equation (5):

$$
\Phi_{1}\left(\vec{x}_{2}\right)=\frac{k e}{\left|\vec{x}_{1}-\vec{x}_{2}\right|}=\frac{k e}{\left|-d \hat{x}-\vec{x}_{2}\right|}=\frac{k e}{\sqrt{d^{2}+\left|\vec{x}_{2}\right|^{2}+2 d x_{2}}} .
$$


From now on we drop the index 2 and use standard spherical coordinates. Hence $r=\left|\vec{x}_{2}\right|$ and $x_{2}=r \sin \theta \cos \varphi$, thus:

$$
\Phi_{1}(\vec{x})=\frac{k e}{\sqrt{d^{2}+r^{2}+2 d r \sin \theta \cos \varphi}} .
$$

the potential $\varphi$ dependence clearly demonstrates the symmetry breaking of the above potential. We can now calculate the relativistic motor momentum defined in Equation (7) by inserting the potential of Equation (76) and the current obtained in Equation (45) for an electron in an Eigen state of the hydrogen atom:

$$
\vec{P}=\frac{\mu_{0}}{4 \pi} e \bar{J} m a_{0} \int_{0}^{\infty} r d r \int_{0}^{\pi} d \theta \int_{0}^{2 \pi} d \varphi \frac{A^{\prime 2}}{\sqrt{d^{2}+r^{2}+2 d r \sin \theta \cos \varphi}}(-\sin \varphi \hat{x}+\cos \varphi \hat{y}) .
$$

in which $\bar{J}$ is given in Equation (51), $m$ is the magnetic quantum number (not the mass), and $\hat{\varphi}$ is given through Equation (47). Finally:

$$
A^{\prime}(r, \theta)=\left|\psi_{n l m}\right| a_{0}^{\frac{3}{2}}
$$

is the dimensionless state amplitude in which we suppress the quantum indices $n l m$. The above expression can be somewhat simplified as follows:

$$
\vec{P}=\frac{\mu_{0}}{4 \pi} e \bar{J} m a_{0} \int_{0}^{\infty} \frac{r d r}{\sqrt{d^{2}+r^{2}}} \int_{0}^{\pi} d \theta A^{\prime 2} \int_{0}^{2 \pi} d \varphi \frac{-\sin \varphi \hat{x}+\cos \varphi \hat{y}}{\sqrt{1+B \cos \varphi}}
$$

in the above:

$$
B(r, \theta, d) \equiv \frac{2 r d \sin \theta}{d^{2}+r^{2}}, \quad 0 \leq B \leq 1 .
$$

Obviously the cylindrical symmetry is restored when $B=0$. This happens for either small $d$ or large $d$, that is if the proton is too close or too far from the electron:

$$
\lim _{d \rightarrow 0} B=\lim _{d \rightarrow \infty} B=0
$$

hence a relativistic motor effect may occur only in intermediate values of $d$. We notice that $\sin \theta \geq 0$ as $\theta \in[0, \pi]$, thus we are only interested in $B \geq 0$. We also notice that the maximal value of $B$ is for $\theta=\frac{\pi}{2}$ and for $r=d$ for which $B_{\max }=1$ as depicted in Figure 2.

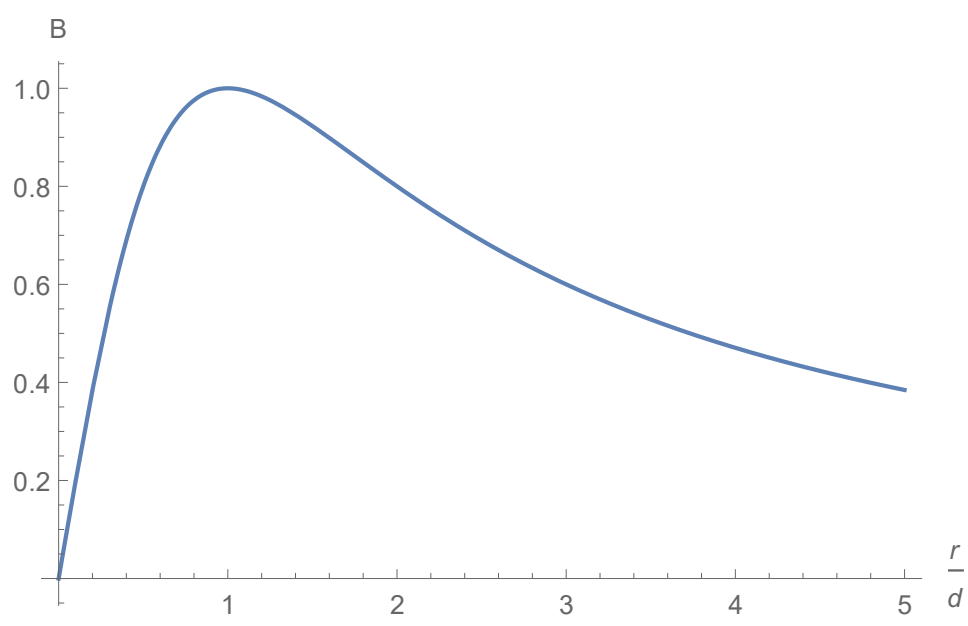

Figure 2. $B$ as function of $\frac{r}{d}$ for $\theta=\frac{\pi}{2}$. 
A triple integral needs to be evaluated in order to calculate the momentum, this cannot be done using only analytic techniques, however, at least part of the integration can be done analytically. Consider the integrals:

$$
I_{x}(B) \equiv \int_{0}^{2 \pi} d \varphi \frac{\sin \varphi}{\sqrt{1+B \cos \varphi}}, \quad I_{y}(B) \equiv \int_{0}^{2 \pi} d \varphi \frac{\cos \varphi}{\sqrt{1+B \cos \varphi}} .
$$

Integrating analytically we arrive at the following results:

$$
I_{x}(B)=0, \quad I_{y}(B)=\frac{4(1+B) \text { Eliptic }_{E}\left(\frac{2 B}{1+B}\right)-4 \text { Elliptic }_{K}\left(\frac{2 B}{1+B}\right)}{B \sqrt{1+B}} .
$$

in the above Elliptic $_{E}$ and Elliptic $_{K}$ are the elliptic $E$ and $K$ functions, respectively. The function $I_{y}$ is an even function that is $I_{y}(B)=I_{y}(-B)$ and is depicted in Figure 3.

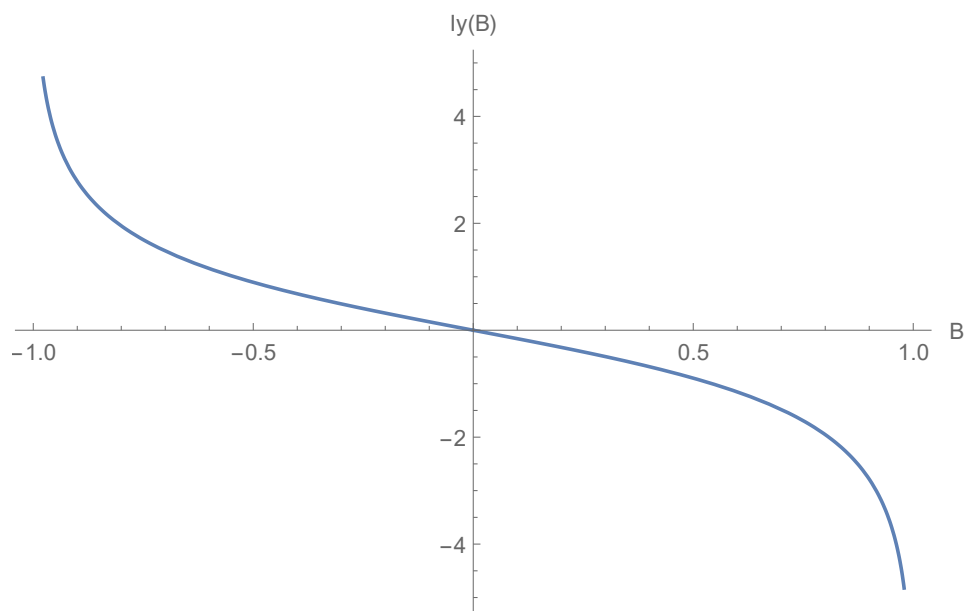

Figure 3. The even function $I_{y}(B)$.

We remark that since $B \geq 0$ it follows that $I_{y}(B) \leq 0$, thus only the right hand part of Figure 3 is of interest. We also notice that $I_{y}(B)$ is monotonic decreasing function of $B$ hence the larger values of $B$ which are around $r=d, \theta=\frac{\pi}{2}$ will make the most significant contribution (in absolute terms) to $I_{y}(B)$. For $B=1$ this function is singular, however, the singularity will be integrated away when integrating over $\theta$.

We have obtained a counter intuitive result that the momentum is generated in the $y$ direction despite the fact that proton which breaks the symmetry is located in the $x$ direction, that is:

$$
\vec{P}=\frac{\mu_{0}}{4 \pi} e \bar{J} m a_{0} \int_{0}^{\infty} \frac{r d r}{\sqrt{d^{2}+r^{2}}} \int_{0}^{\pi} d \theta A^{\prime 2} I_{y}(B) \hat{y} .
$$

Next we define the function $F$ as follows:

$$
F \equiv \int_{0}^{\pi} d \theta A^{\prime 2} I_{y}(B)
$$

$F$ will become larger if both $A^{\prime 2}$ and $I_{y}$ are peaked at the same $r$ value, if the overlap is small so will be the relativistic momentum. We can thus write the momentum $\vec{P}$ in the form:

$$
\vec{P}=\frac{\mu_{0}}{4 \pi} e \bar{J} m a_{0} \int_{0}^{\infty} \frac{r d r}{\sqrt{d^{2}+r^{2}}} F \hat{y}=\frac{\mu_{0}}{4 \pi} e \bar{J} \frac{a_{0}^{3}}{d} m \int_{0}^{\infty} F \frac{\left(\frac{r}{a_{0}}\right) d\left(\frac{r}{a_{0}}\right)}{\sqrt{1+\left(\frac{r}{d}\right)^{2}}} \hat{y} .
$$

Thus, $\vec{P}$ is given in the form:

$$
\vec{P}=\bar{P} \tilde{P} \hat{y}
$$


in which $\bar{P}$ is a dimensional constant independent of the quantum state of the electron:

$$
\bar{P}=\frac{\mu_{0}}{4 \pi} e \bar{J} \frac{a_{0}^{3}}{d} .
$$

Furthermore, $\tilde{P}$ is dimensionless, and depends on the quantum state.

$$
\tilde{P}_{n l m}=m \frac{n^{2}}{4} \int_{0}^{\infty} F_{n l m} \frac{r^{\prime} d r^{\prime}}{\sqrt{1+\left(\frac{r^{\prime}}{d^{\prime}}\right)^{2}}} .
$$

in the above we have made explicit the dependence on the quantum numbers and have made use of the normalized $r^{\prime}$ defined in Equation (41) and also introduced a normalized $d^{\prime}$ using a similar definition:

$$
d^{\prime} \equiv \frac{2 d}{n a_{0}} .
$$

We also notice that since the hydrogen Eigen state is given as a function of $r^{\prime}$ and also $B$ being dimensionless can be written in terms of dimensionless quantity:

$$
B=\frac{2 r^{\prime} d^{\prime} \sin \theta}{d^{\prime 2}+r^{\prime 2}} .
$$

it follows that $\tilde{P}_{n l m}$ only depends on the quantum state and $d^{\prime}$.

We shall now investigate the case $d=2 a_{0}$. In such a case the Hamiltonian of the electron is modified significantly and thus will have different Eigenstates from the ones that are obtained for an isolated hydrogenatom. Hence the Eigen states described in Equation (38) can only be considered as a superposition of the true Eigen states. Nevertheless, it will suffice to study the relativistic momentum generated by the hydrogenEigen states in this preliminary study. In this case:

$$
\bar{P}=\frac{\mu_{0}}{8 \pi} e \bar{J} a_{0}^{2} \simeq 5.310^{-29} \mathrm{Kg} \mathrm{m} / \mathrm{s}
$$

The velocity associate withe this momentum is:

$$
\bar{v}=\frac{\bar{P}}{2 m_{p}} \simeq 0.016 \mathrm{~m} / \mathrm{s},
$$

in we have taken into account both the mass of the proton and the mass of the hydrogenatom. Hence for an unloaded relativistic motor we will obtain a velocity of:

$$
\vec{v}=\frac{\vec{P}}{2 m_{p}} \simeq 0.016 \tilde{P} \hat{y} \mathrm{~m} / \mathrm{s} .
$$

We shall look at two cases of excited states.

\subsubsection{The Case 211}

In the low excited state $n=2, l=1, m=1$ the square amplitude $A_{211}^{\prime 2}$ is depicted in Figure 4. 


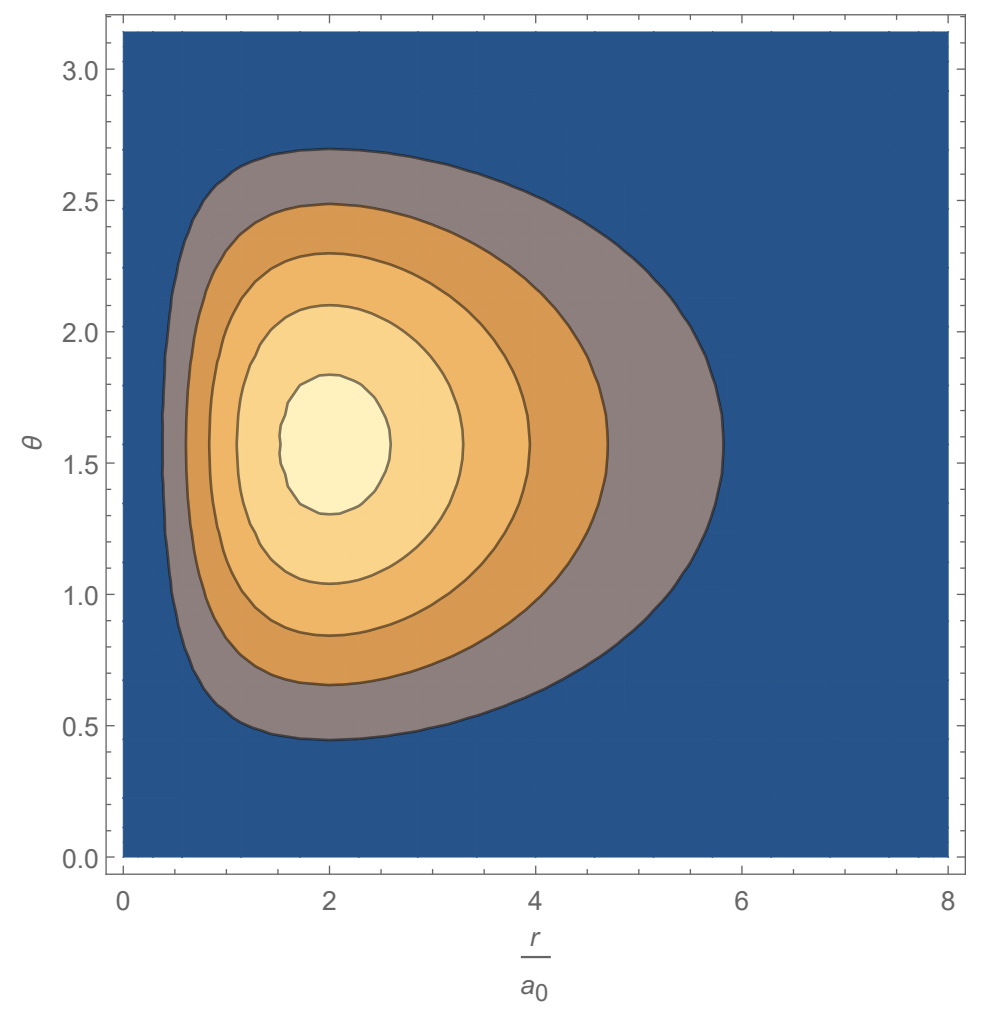

Figure 4 . The square amplitude $A_{211}^{\prime 2}$.

It is seen that the function describes torii of equi-value. The function peaks at $\theta=\frac{\pi}{2}$ at $r=2 a_{0}$. A cross section of the same for $\theta=\frac{\pi}{2}$ is described in Figure 5:

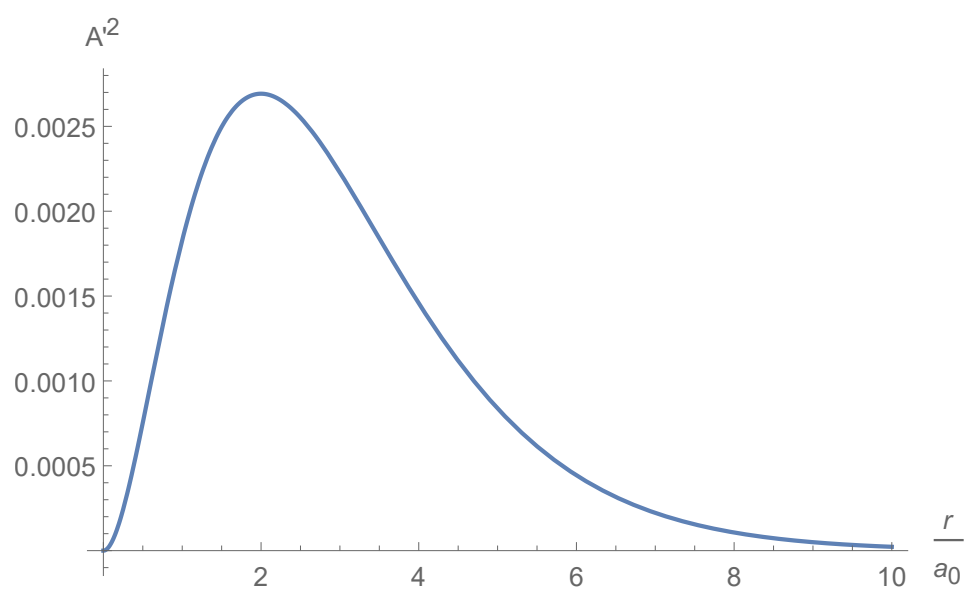

Figure 5. The square amplitude $A_{211}^{\prime 2}$ at $\theta=\frac{\pi}{2}$.

We are now able to evaluate numerically $F_{211}$ defined in Equation (85) which is depicted in Figure 6. 


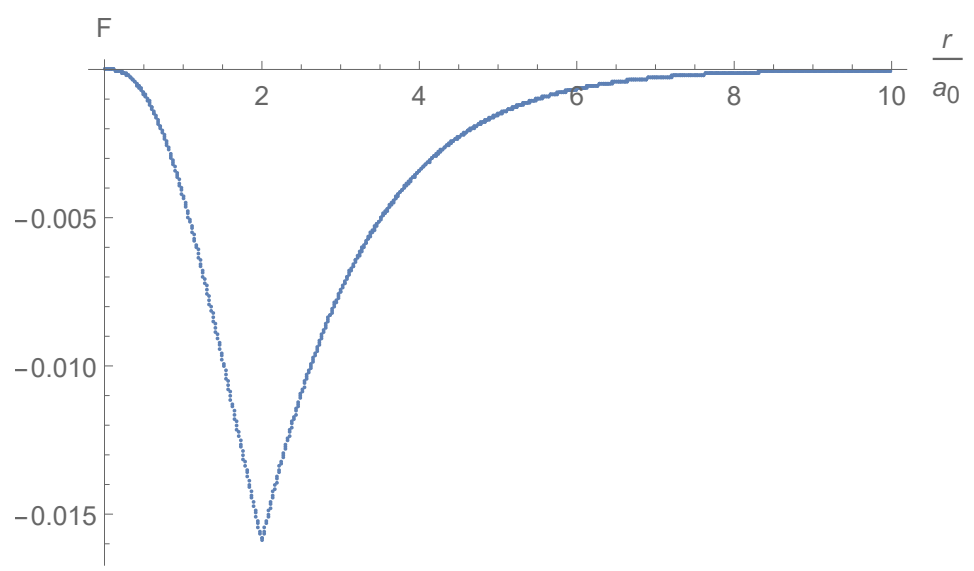

Figure 6. The function $F_{211}$.

Finally using $F_{211}$ we can calculate numerically $\tilde{P}_{211}$ using Equation (89) and obtain the value:

$$
\tilde{P}_{211} \simeq-0.047
$$

Hence according to Equations (87) and (94) we obtain:

$$
\vec{P}_{211} \simeq-2.4710^{-30} \hat{y} \mathrm{~kg} \mathrm{~m} / \mathrm{s} \quad \vec{v}_{211}=\simeq-7.410^{-4} \hat{y} \mathrm{~m} / \mathrm{s}
$$

Obviously even if many such systems are accumulated the total mass will grow accordingly and the final achievable velocity will be rather slow.

\subsubsection{The Case 433}

In the high excited state $n=4, l=3, m=3$ the square amplitude $A_{433}^{\prime 2}$ is depicted in Figure 7.

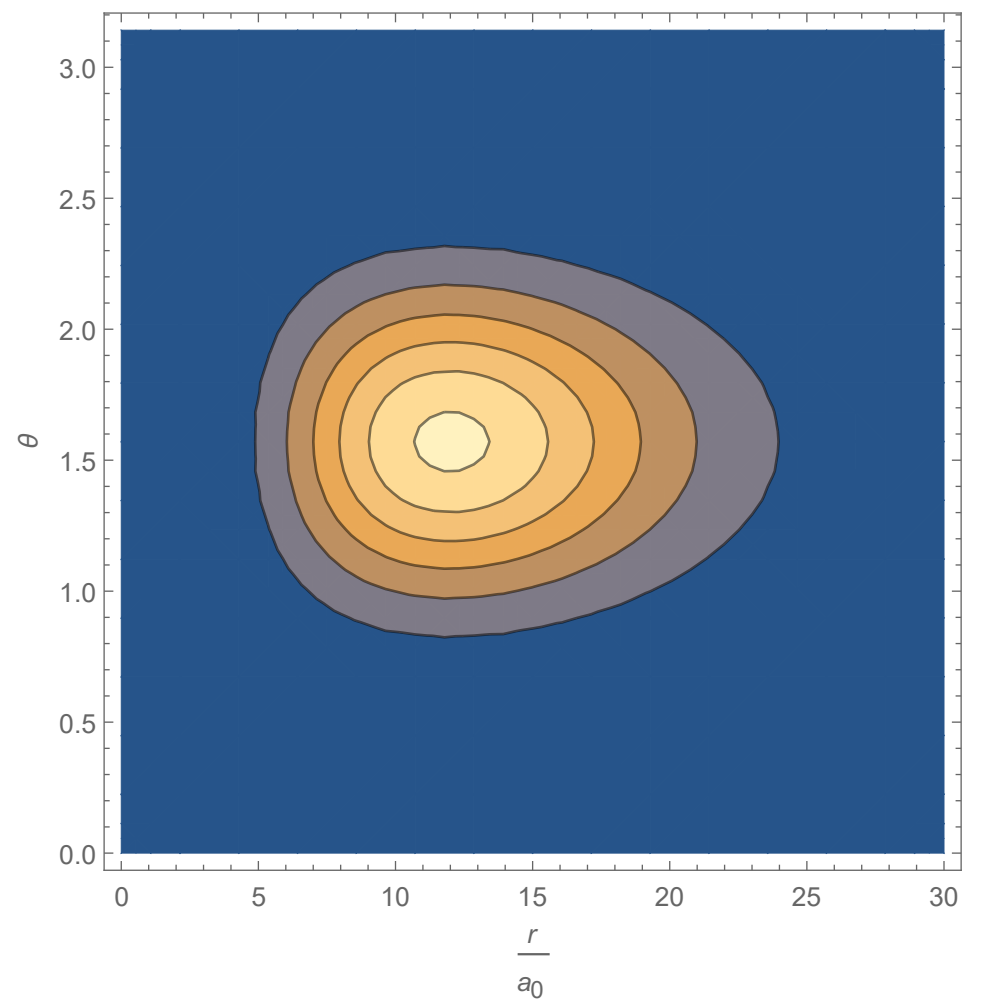

Figure 7. The square amplitude $A_{433}^{\prime 2}$. 
It is seen that the function describes torii of equi-value. The function peaks at $\theta=\frac{\pi}{2}$ at $r \simeq 12 a_{0}$, hence the hydrogenatom becomes much larger. A cross section of the same for $\theta=\frac{\pi}{2}$ is described in Figure 8:

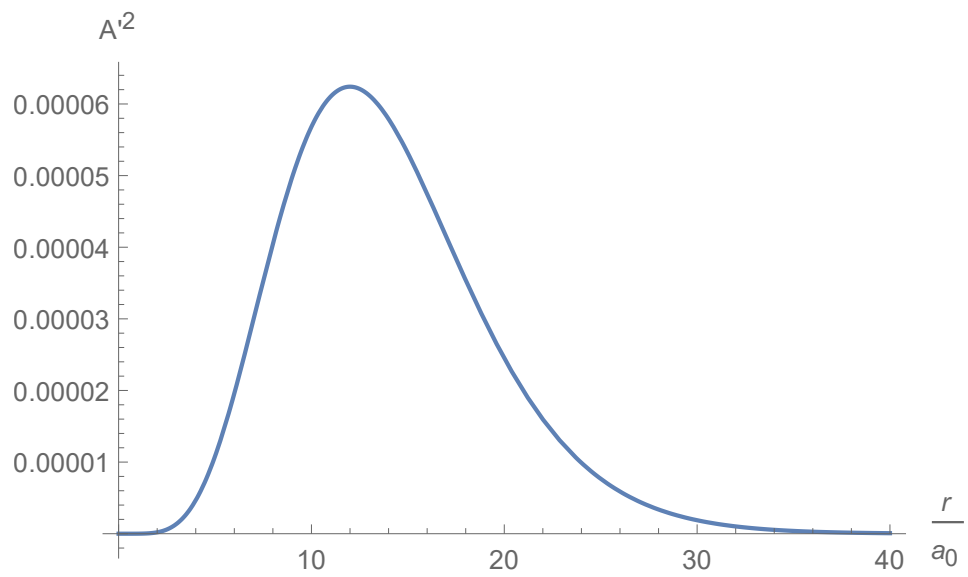

Figure 8. The square amplitude $A_{433}^{\prime 2}$ at $\theta=\frac{\pi}{2}$.

We are now able to evaluate numerically $F_{433}$ defined in Equation (85) which is depicted in Figure 9.

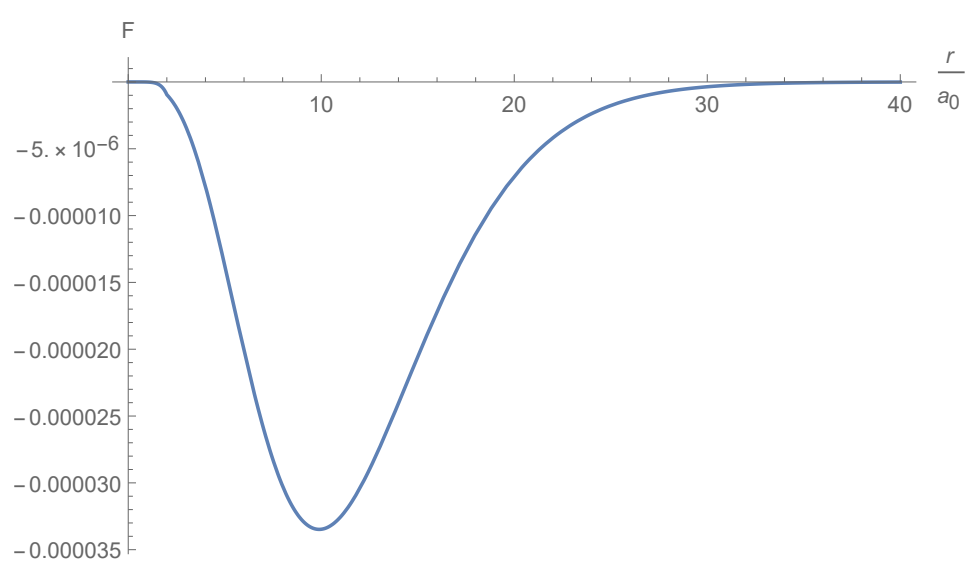

Figure 9. The function $F_{433}$.

Finally using $F_{433}$ we can calculate numerically $\tilde{P}_{433}$ using Equation (89) and obtain the value:

$$
\tilde{P}_{433} \simeq-0.002
$$

this is surprisingly smaller than $P_{211}$, but can be understood due to the small overlap between $I_{y}(B)$ and $A_{433}^{\prime 2}$. Now according to Equations (87) and (94) we obtain:

$$
\vec{P}_{433} \simeq-1.0510^{-31} \hat{y} \mathrm{~kg} \mathrm{~m} / \mathrm{s} \quad \vec{v}_{433}=\simeq-3.110^{-5} \hat{y} \mathrm{~m} / \mathrm{s} .
$$

Obviously even if many such systems are accumulated the total mass will grow accordingly and the final achievable velocity will be rather slow. The situation can be some what improved if the overlap between $I_{y}(B)$ and $A_{433}^{\prime 2}$ is better. This will happen if we choose $d \simeq 12 a_{0}$, however, a detailed calculation gives in this case:

$$
\vec{P}_{433} \simeq-2.710^{-31} \hat{y} \mathrm{~kg} \mathrm{~m} / \mathrm{s} \quad \vec{v}_{433}=\simeq-8.010^{-5} \hat{y} \mathrm{~m} / \mathrm{s} .
$$

this is better than before but worse than what is obtained in a low excited state 211 . 


\subsubsection{A Nano Relativistic Motor}

A similar analysis was attempted for a very high excited state $n=11, l=10, m=10$, in this case which appears in Humphrey's series [27] $A_{11}^{\prime 2} 10{ }_{10}$ peaks at $110 a_{0} \simeq 5.8$ nano m, which thus justly deserves the name a nano relativistic motor. Choosing $d$ to coincide with the above value we obtain, however, after a detailed calculation the values:

$$
\vec{P}_{111010} \simeq-2.010^{-32} \hat{y} \mathrm{~kg} \mathrm{~m} / \mathrm{s} \quad \vec{v}_{111010}=\simeq-5.910^{-6} \hat{y} \mathrm{~m} / \mathrm{s} .
$$

this does not qualify as an improvement.

\section{Discussion}

In a previous paper (and in earlier papers of other authors), it was demonstrated that Newton's third law is not compatible with the principles of special relativity and thus the total force on a general two charged body system is not null. Momentum is, however, conserved if one does not neglect the field momentum, and the same can be said about the energy.

The dielectric strength's and current density limitations of macroscopic bodies led us to consider the microscopic realm in which charge densities and current densities are considerably higher.

The main results of this paper are the possibility of implementing a relativistic motor in the atomic and nano scales. It is shown that a hydrogen atom whether in a ground or excited state does not produce any momentum according to the relativistic motor equation.

Thus, two configurations are studied, in one the Hydrogen electron is put in a wave packet state rather than in an Eigen state. In this approach one can reach high momentum which means high velocity for both a loaded and an unloaded engine. However, for a considerable momentum to be realized the wave packet must be highly localized to a subatomic scale. To achieve even higher momentum, sub nuclear scales are required. In order to change the state of hydrogen atom from the ground state to any desired state a suitable Hamiltonian is to be constructed. Thus, the desired wave packet can only be achieve by an appropriate electromagnetic field which must be non trivial.

A second configuration involves symmetry breaking by adding an additional proton to the hydrogen atom. For simplicity we assume that despite this addition the Eigenstates of the hydrogen atom are still valid. This approach yields low momenta and low velocities for high and low excited states.

\section{Conclusions}

To conclude we remark that despite the possibility in principle to construct a working relativistic motor, this is not a trivial task and involves the creation of a highly localized wave packet. Thus, in a study which is not a merely preliminary as this one, the electromagnetic field needed to achieve this goal must be specified. Furthermore, we notice that the wave packet should not only be created but also maintained for an engine that may become useful.

Additional directions for future studies which are arise from this paper include:

1. The analysis of a relativistic motor of which its components move also at relativistic speeds and not just the electromagnetic signals transmitted between the components. The need for this arises as the electron studied in the current paper moves at relativistic speeds.

2. For the same reason an analysis of the relativistic motor in the frame work of a Dirac theory is required. The Schrödinger equation and even the Pauli equation are not appropriate for the study of an electron at relativistic speeds.

3. For the symmetry breaking approach, despite the fact that it shows little promise for a useful relativistic motor, one should use true Eigenstates (and not Eigenstates of the hydrogen atom which are clearly not appropriate). Perhaps for those Eigenstates the results will be more encouraging.

Funding: This research received no external funding.

Institutional Review Board Statement: Not applicable. 
Informed Consent Statement: Not applicable.

Conflicts of Interest: The author declares no conflict of interest.

\section{References}

1. Einstein, A. On the Electrodynamics of Moving Bodies. Annalen der Physik 1905, 17, 891-921. [CrossRef]

2. Maxwell, J.C. A dynamical theory of the electromagnetic field. Philos. Trans. R. Soc. Lond. 1865, 155, 459-512.

3. Jackson, J.D. Classical Electrodynamics, 3rd ed.; Wiley: New York, NY, USA, 1999.

4. $\quad$ Feynman, R.P.; Leighton, R.B.; Sands, M.L. Feynman Lectures on Physics; Revised 50th Anniversary Edition; Basic Books: New York, NY, USA, 2011.

5. Heaviside, O. On the Electromagnetic Effects due to the Motion of Electrification through a Dielectric. Philos. Mag. 1889, 27, 324-339. [CrossRef]

6. Newton, I. Philosophiae Naturalis Principia Mathematica; Jussu Societatis Regiae ac typis Iosephi Streater, Prostat apud Plures Bibliopolas: London, UK, 1687.

7. Goldstein, H.; Poole, C., Jr.; Safko, J.L. Classical Mechanics, 3rd ed.; Pearson: London, UK, 2001.

8. D'Abramo, G. On apparent faster-than-light behavior of moving electric fields. Eur. Phys. J. Plus 2021, 136, 301. [CrossRef]

9. Rajput, S.; Yahalom, A. Newton's Third Law in the Framework of Special Relativity for Charged Bodies. Symmetry 2021, 13, 1250. [CrossRef]

10. Tuval, M.; Yahalom, A. Momentum Conservation in a Relativistic Engine. Eur. Phys. J. Plus 2016, 131, 374. [CrossRef]

11. Mansuripur, M. Trouble with the Lorentz Law of Force: Incompatibility with Special Relativity and Momentum Conservation. Phys. Rev. Lett. 2012, 108, 193901. [CrossRef] [PubMed]

12. Griffiths, D.J.; Heald, M.A. Time dependent generalizations of the Biot-Savart and Coulomb laws. Am. J. Phys. 1991, 59, 111-117. http:/ /dx.doi.org/10.1119/1.16589. [CrossRef]

13. Jefimenko, O.D. Electricity and Magnetism, Appleton-Century Crofts, New York (1966), 2nd ed.; Electret Scientific: Star City, WV, USA, 1989.

14. Tuval, M.; Yahalom, A. Newton's Third Law in the Framework of Special Relativity. Eur. Phys. J. Plus 2014, 129, 240. [CrossRef]

15. Yahalom, A. Retardation in Special Relativity and the Design of a Relativistic Motor. Acta Phys. Pol. A 2017, 131, 1285-1288. [CrossRef]

16. Yahalom, A. Preliminary Energy Considerations in a Relativistic Engine. In Proceedings of the Israeli-Russian Bi-National Workshop "The Optimization of Composition, Structure and Properties of Metals, Oxides, Composites, Nano- and Amorphous Materials", Ariel, Israel, 28-31 August 2017; pp. 203-213.

17. Rajput, S.; Yahalom, A. Material Engineering and Design of a Relativistic Engine: How to Avoid Radiation Losses. Adv. Eng. Forum 2020, 36, 126-131. [CrossRef]

18. Rajput, S.; Yahalom, A.; Qin, H. Lorentz Symmetry Group, Retardation and Energy Transformations in a Relativistic Engine. Symmetry 2021, 13, 420. [CrossRef]

19. Dielectric Strength of Air-The Physics Fact Book. Available online: https://hypertextbook.com/facts/2000/AliceHong.shtml (accessed on 18 November 2021).

20. Giere, S.; Kurrat, M.; Schümann, U. HV Dielectric Strength of Shielding Electrodes in Vacuum Circuit-Breakers. In Proceedings of the 20th International Symposium on Discharges and Electrical Insulation in Vacuum, Tours, France, 30 June-5 July 2002.

21. Gabrysch, M. Electronic Properties of Diamond. Available online: el.angstrom.uu.se (accessed on 10 August 2013).

22. Hsi-wen, L.; Yu-Chong, T. Parylene-based electret power generators. J. Micromech. Microeng. 2008, 18, 104006.

23. Jung, S.G.;Kang, J.H.; Park, E.; Lee, S.; Lin, J.Y.; Chareev, D.A.; Vasiliev, A.N.; Park, T. Enhanced critical current density in the pressure-induced magnetic state of the high-temperature superconductor FeSe. Sci. Rep. 2015, 5, 16385. [CrossRef] [PubMed]

24. Peebles, P.Z. Probability, Random Variables and Random Signal Principles; McGraw Hill: New York, NY, USA, 2001.

25. Schrödinger, E. Collected Papers in Wave Mechanics; Blackie and Sons: London, UK, 1928; p. 102.

26. Holland, P.R. The Quantum Theory of Motion; Cambridge University Press: Cambridge, UK, 1993.

27. Humphreys, C.J. The Sixth Series in the Spectrum of Atomic Hydrogen. J. Res. Natl. Bur. Stand. 1953, 50, 1. [CrossRef] 